\title{
EZH2 inhibitors-mediated epigenetic reactivation of FOSB inhibits triple-negative breast cancer progress
}

\author{
Ruishan Zhang, Xiang Li, Zhuangkai Liu, Yuying Wang, Hao Zhang and Hong Xu* ${ }^{*}$
}

\begin{abstract}
Background: Triple-negative breast cancer (TNBC) is the most aggressive subtype of breast cancer that lacks expression of estrogen receptor (ER) and progesterone receptor (PR) and the human epidermal growth factor receptor 2 (HER2) gene. Chemotherapy remains the standard of care for TNBC treatment, but considerable patients are very resistant to chemotherapy. Mutations or aberrant upregulation of EZH2 occur frequently, and EZH2 inhibitor (EZH2i) showed some preclinic antitumor effects in TNBC.

Methods: RNA-seq data of 3 TNBC cell lines either treated with 2 HM GSK343, or stably transduced with shEHZ2, compared to untreated controls (GSE112378) were analyzed by Limma R package. The Kaplan-Meier plotter (KM plotter) database was used to assess the relevance of FOSB mRNA expression to relapse-free survival (RFS) in TNBC. Cell number counting and colony formation assays were used to detect the biological effect of FOSB on the growth of TNBC cells in vitro. The effect of FOSB on TNBC tumor growth in vivo was investigated in a mice tumor xenograft model. Luciferase reporter and chromatin immunoprecipitation (Chip) assays were used to determine the regulatory roles of C/EBPß on FOSB expression.

Results: We found that FOSB, a member of the activator protein-1 complex, was a direct downstream target of EZH2. FOSB was significantly decreased in TNBC samples and associated with better relapse-free survival (RFS). EZH2-mediated histone 3 trimethylated on lysine 27 (H3K27me3), a marker of silent chromatin conformation, at the FOSB promoter inhibited it expression. Depletion of FOSB in TNBC cells promoted cell proliferation in vitro and tumor growth in vitro by inactivating the p53 pathway and conferred resistant to EZH2 inhibitor (EZH2i). Mechanistically, EZH2i promotes the shift from H3K27me3 to H3K27ac at the FOSB promoter, and recruits the transcription factor C/ EBP $\beta$ to activate FOSB gene transcription.
\end{abstract}

Conclusion: Together, our results suggest that EZH2-mediated epigenetic inactivation of FOSB promotes TNBC expression and demonstrate that reactivation of FOSB expression by C/EBP $\beta$ underlies the anti-TNBC action of EZH2is. Keywords: TNBC, EZH2, FOSB, C/EBPß, Epigenetic inactivation

*Correspondence: xuhong4015@yeah.net

Department of Breast Cancer, Cancer Hospital of China Medical University, Liaoning Cancer Hospital and Institute, 44 Xiaoheyan Road, Dadong District, Shenyang 110042, Liaoning, People's Republic of China

\begin{abstract}
Background
Breast cancer is a heterogeneous disease that differs in morphology, molecular biology, clinical manifestations, and responsiveness to treatment [1]. In 2016, approximately 300,000 women were diagnosed with breast cancer and $\sim 30,000$ died (American Cancer Society: Breast cancer facts and figures 2015-2016). Based on the molecular phenotype of breast cancer, it is generally divided
\end{abstract}


into four subtypes: the mammary glandular type, the human epidermal growth factor-2 overexpression type, the normal cell-like type, and the basal-like type [2]. Triple-negative breast cancer (TNBC) is an aggressive basal-like subtype that constitutes $12-18 \%$ of breast cancer patients and frequently develops resistance to chemotherapy [3]. TNBC patients lack the estrogen receptor (ER), progesterone receptor (PR) and HER2 receptor and therefore are not eligible for hormone or anti-Her2 therapy. The lack of targeted therapies and the poor prognosis of patients with TNBC warrants novel molecular targets to treat these patients [4]. Different strategies were used to treatment breast cancer in preclinical models [5-7]. In recent years, epigenetic mechanisms have been discovered to play an important role in the development of TNBC and targeting epigenetic enzymes might represent a novel treatment for TNBC patient $[8,9]$.

EZH2 is the enzymatically active core subunit of the PRC2 complex, which contains EED, SUZ12, and $\mathrm{RbAp} 46 / 48$. PRC2 methylates the lysine residue at position 27 of histone 3 (H3K27me3) to facilitate chromatin compaction and gene silencing [10, 11]. EZH2 was firstly identified as an oncogene in prostate and breast cancer, which is highly expressed in hormone-resistant and metastatic prostate cancers and TNBC with lower overall survival $[12,13]$. Subsequent research found that EZH2 was highly expressed in a variety of malignancies, including head and neck cancer, bladder cancer, colorectal cancer, and non-small cell lung cancer, and was associated with poor prognosis [10]. High levels of EZH2 were shown to correlate with aggressiveness and advanced disease in each of these cancer types [10]. EZH2 has been shown to be essential for proliferation of cancer cell lines that is largely dependent upon its methyltransferase domain [14]. Forced expression of EZH2 leads to neoplastic transformation of breast epithelial cells and development of myeloproliferative disorder in mice [15]. Recurrent heterozygous point mutations at tyrosine 641 (Y641) within the C-terminal catalytic SET domain of EZH2 occur in 22\% of germinal center B-cell (GCB) diffuse large cell B-cell lymphomas (DLBCL) and in 7\% to $12 \%$ of follicular lymphomas (FL) $[16,17]$. The mutant EZH2 caused aberrant H3K27me3 that leaded to repression of Polycomb target genes. Given the evidence for EZH2 enzymatic gain-of-function being a cancer driver, development of specific EZH2 inhibitors (EZH2is) has received widespread attention [18]. The existing preclinical research evidences show that EZH2is only have a certain therapeutic effect on individual hematological tumors containing EZH2 activating mutations [19]. For example, two EZH2 inhibitors, GSK343 and EPZ-6438 demonstrated preliminary benefits in DLBCL and FL $[20,21]$. However, EZH2is are basically ineffective in the treatment of solid tumors, which greatly limits the clinical application of these inhibitors. A recent study found that EZH2is can inhibit H3K27me3 while promoting H3K27ac, resulting in epigenetic reactivation of oncogenic genes transcription [22]. Therefore, the identification of the target genes and downstream signals that were abnormally activated after EZH2is treatment might be beneficial for clarifying the causes of EZH2is resistance in solid tumor cells.

AP-1 transcription factor consists of a variety of dimers from the members of the JUN proto-oncogene (c-JUN) family (c-JUN, JUNB, JUND) or FBJ murine osteosarcoma viral oncogene homolog (FOS) family (c-FOS, FOSB, FRA1/2) [23]. AP-1 has been implicated in lots of biological processes including cell proliferation, death, differentiation and oncogenic transformation [24, 25]. For example, c-FOS could promote the cancer stem-like cell properties in head and neck squamous cell carcinoma [26]. However, the role of FOSB in TNBC has not been fully revealed. In this study, we identified FOSB as a novel EZH2 downstream target gene in TNBC, and inhibition of which resulted in tumor growth and EZH2is resistance.

\section{Materials and methods \\ Clinical samples and data acquisition}

Transcriptome RNA-sequencing (RNA-seq, FPKM) data of TNBC were downloaded from the TCGA data portal (https://cancergenome.nih.gov/), which contained data from 115 primary TNBC and 113 non-tumor tissues. RNA-seq data (RPKM) of 3 TNBC cell lines either treated with $2 \mu \mathrm{M}$ GSK343, or stably transduced with shEHZ2, compared to untreated controls (GSE112378) were used for analysis by the R software Linear Models for Microarray and RNA-Seq Data (Limma) package (http://bioco nductor.org/packages/Limma/). We performed differential gene analysis of all transcriptional data, setting a $\log 2$ |fold change $\mid>1$ and a false discovery rate (FDR) $<0.05$ as the cutoff values. The Wilcox-test was used for analyses. In 115 cases of TNBC RNA-Seq data (FPKM), FOSB was analyzed by GSEA 4.0.1 (with h.all.v7.0.symbols.gmt as the background gene set).

\section{Cell lines and reagents}

Breast cancer cell lines MDA-MB-231 and MDA-MB-436 cells were purchased from Cell lines Cell Bank of Chinese Academy of Sciences; HEK293T cells were kept in our lab for routine work.

Cells were cultured in Dulbecco's modified Eagle Medium (DMEM; Gibco BRL, Grand Island, NY, USA) with 10\% FBS (Gibco, Gaithersburg, MD, USA), 100 U/ $\mathrm{ml}$ penicillin and $100 \mu \mathrm{g} / \mathrm{ml}$ streptomycin, and maintained at $37{ }^{\circ} \mathrm{C}$ in a humidified chamber $\left(5 \% \mathrm{CO}_{2}\right)$. All 
cell lines were routinely tested negative for Mycoplasma. GSK343 was purchased from Medchemexpress (HY13500). Cycloheximide (239764) was purchased from Calbiochem (San Diego, CA, USA). The proteasome inhibitor MG132 was purchased from Sigma-Aldrich (St. Louis, MO, USA).

\section{RNA isolation, siRNAs and real-time PCR}

Total RNAs from cells were extracted by using Trizol reagents (Invitrogen, Shanghai). The mRNAs were then reversed transcribed into complementary DNA (cDNA) using the Promega Reverse Transcription System (Madison, WI, USA). Oligo dT was used to prime cDNA synthesis. Real-time PCR was performed using a SYBR Green Premix Ex Taq (Takara, Japan) on a Light Cycler 480 (Roche, Switzerland).The mRNA levels of GAPDH were used as internal control. Differences in gene expression were calculated using the $2-\Delta \Delta \mathrm{Ct}$ method and expressed as fold-changes. PCR conditions included an initial holding period at $95^{\circ} \mathrm{C}$ for $5 \mathrm{~min}$, followed by a two-step PCR program consisting of $95{ }^{\circ} \mathrm{C}$ for $5 \mathrm{~s}$ and $60{ }^{\circ} \mathrm{C}$ for $30 \mathrm{~s}$ for 50 cycles. Primers used for qPCR analysis were list as follows: FOSB forward, 5'-GCTGCAAGA TCCCCTACGAAG-3'; reverse, 5'-ACGAAGAAGTGT ACGAAGGGTT3'; EZH2 forward, 5'-AATCAGAGT ACATGCGACTGAGA-3'; reverse, 5'-GCTGTATCC TTCGCTGTTTCC; TP53 forward, 5'-CAGCACATG ACGGAGGTTGT-3'; reverse, 5'-TCATCCAAATAC TCCACACGC-3'; CDKN1(p21 ${ }^{\text {CIP1 }}$ )forward, $5^{\prime}$-TGT CCGTCAGAACCCATGC-3'; reverse, 5'-AAAGTC GAAGTTCCATCGCTC-3'; GAPDH forward, 5'- GGA GCGAGATCCCTCCAAAAT-3'; reverse, 5'-GGCTGT TGTCATACTTCTCATGG-3'. For knockdown experiments, cells were transiently transfected by siRNA pools with TransIT-X2 transfection reagent (Mirus, Madison, WI). Control-siRNAs were from Santa Cruz (sc-37007). EZH2-siRNAs were from Cell Signaling Technology (6509), CEBPB-siRNAs were from Santa Cruz (sc-29229) and SUZ12-siRNA (h) were from Santa Cruz (sc-45597).

\section{Western blotting}

Cells were lysed with lysis buffer $(100 \mathrm{mM}$ Tris- $\mathrm{HCl}, \mathrm{pH}$ 6.8, $100 \mathrm{mM}$ DTT, 1\% SDS, $10 \%$ glycerol). Proteins were separated by $10-12 \%$ SDS-PAGE, and transferred to PVDF membranes. Membranes were blocked in 5\% nonfat milk in phosphate-buffered saline (PBS) for $1 \mathrm{~h}$ before incubation with primary antibody overnight at $4{ }^{\circ} \mathrm{C}$. Membranes were washed and blocked with $5 \%$ milk and incubated with different primary antibodies overnight at $4{ }^{\circ} \mathrm{C}$, followed by incubation with secondary antibodies. The primary antibodies used in western blotting included anti-Fos B(F-7) (sc-398595; Santa Cruz; 1:1000 dilution), anti-CEBPB (sc-7962; Santa Cruz; 1:1000 dilution), anti- histone H3K27me3 (ab6002, Abcam; 1:1000 dilution), anti-histone H3K27ac (\#8173, Cell Signaling; 1:1000 dilution) and anti-GAPDH (sc-47724; Santa Cruz; 1:5000).

\section{Luciferase reporter and chromatin immunoprecipitation (ChIP)}

The promoter region of FOSB gene was amplified from the genomic DNA of 293T cells and inserted into pGL4.15 vector (Promega, Madison, Wisconsin, USA). For the luciferase reporter assays, HEK293T cells were seeded in 24-well plates and transfected with the indicated plasmids using Lipo2000 for $36 \mathrm{~h}$. Luciferase activity was measured using the Dual Luciferase Reporter Assay System (Promega). The firefly luciferase luminescence data were normalized by the Renilla luciferase luminescence data. A chromatin immunoprecipitation assay kit was used (Millipore, USA). In brief, cells fixed with $1 \%$ formaldehyde (Sigma, USA) and harvested in SDS lysis buffer. DNA was sheared to fragments of 200-1000 bp by sonications. Lysates containing soluble chromatin were incubated and precipitated overnight with $2 \mu \mathrm{g}$ of anti- histone H3K27me3 (ab6002, Abcam), anti-histone H3K27ac (\#8173, Cell Signaling), anti-EZH2 (E7031, Sigma-Aldrich); anti-C/EBP $\beta$ (sc-7962, Santa Cruz) or rabbit IgG (\#ab172730, Abcam). Protein G agarose was then added for $4 \mathrm{~h}$. Protein-DNA crosslinks were reversed by treatment with proteinase $K$ for $2 h$ at $45^{\circ} \mathrm{C}$. The DNA was subsequently purified, diluted and subjected in the quantitative real-time PCR reactions.

\section{Construction of stable cell line}

HEK293T cells were co-transfected with the vector control or pBabe-FOSB plasmids and packaging vectors for $48 \mathrm{~h}$. Filtered viral supernatants were then collected and added to MDA-MB-436 cells with $10 \mu \mathrm{g} / \mathrm{ml}$ Ploybrane for $48 \mathrm{~h}$ and selected with puromycin $(2 \mu \mathrm{g} / \mathrm{ml})$ for 2 weeks.

\section{CRISPR/Cas9 knock out (KO) cell lines}

The FOSB knock-out MDA-MB-231 cells were generated by CRISPR/Cas9 technology [27]. Briefly, single-stranded DNA oligonucleotides targeted to the coding sites of the gene was designed according to the Crisprgold website (https://crisprgold.mdc-berlin.de/index.php). Annealed primers were cloned into the vector PX459. Primers used for FOSB were list: Forward primer: CACCGTCGTAGG GGTCGACGACCGG). Reverse primer: AAACCCGGT CGTCGACCCCTACGAC). MDA-MB-231 cells were transfected with PX459-FOSB plasmid using Lipo2000 following the manufacturer's instructions. Cells were selected with $2 \mu \mathrm{g} / \mathrm{ml}$ puromycin for about 2 weeks. Single clones were then selected and the knockout efficiency 
was verified by western blot assay with anti-FOSB antibody.

\section{Cell colony formation assay}

Cells were counted, plated in triplicate at a density of 1000 per well in 6-well plates, and cultured in complete medium for about 2 weeks. Then, the cells were washed with PBS and fixed in methanol and stained with crystal violet. The numbers of colonies were then counted.

\section{Xenograft assays}

Animal study was approved by the Animal Care and Use Committee of Liaoning Cancer Hospital and Institute. Four-week-old female BALB/cA nude mice were purchased from National Rodent Laboratory Animal Resources (Shanghai, China). All mice were kept in a specific pathogen-free facility. $1 \times 10^{7}$ FOSB WT or KO MDA-MB-231 cells were suspended in $50 \mu \mathrm{l}$ of DMEM medium, mixed 1:1 with matrigel and injected into the flanks of female nude mice. Tumor sizes were measured by a caliper and calculated using the formula length $\times$ width $2 \times 1 / 2$. Tumor weights were measured after mice were sacrificed.

\section{Statistical analyses}

All experiments were at least repeated three times. Data are presented as mean \pm standard deviation (SD). Results were analyzed using either two-tailed Student's $t$ test or two-way analysis of variance (ANOVA) in Graphpad Prism 7.0 software to assess statistical significance. $\mathrm{P}<0.05$ were considered statistically significant. Statistical significance is displayed as ${ }^{*} \mathrm{P}<0.05,{ }^{* *} \mathrm{P}<0.01$, or **: $\mathrm{P}<0.001$.

\section{Results}

Genetic ablation or pharmacological inhibition of EZH2 resulted in FOSB gene expression in TNBC cells

To identify novel targets of EZH2 in TNBC, we firstly reanalyzed the RNA sequencing analysis (RNA-seq) data of 3 TNBC cell lines either treated with $2 \mu \mathrm{M}$ GSK343, or stably transduced with shEHZ2, compared with untreated controls (GSE112378) [28]. We assumed that genetic ablation or pharmacological inhibition of EZH2 might cause the increase the expression of EZH2 target genes. The Limma $\mathrm{R}$ package identified 20 common differentially expressed genes which were selected to draw a heat map (Fig. 1a). Among the up-regulated genes, we are particularly interested in FOSB, a member of the activator protein-1 complex.

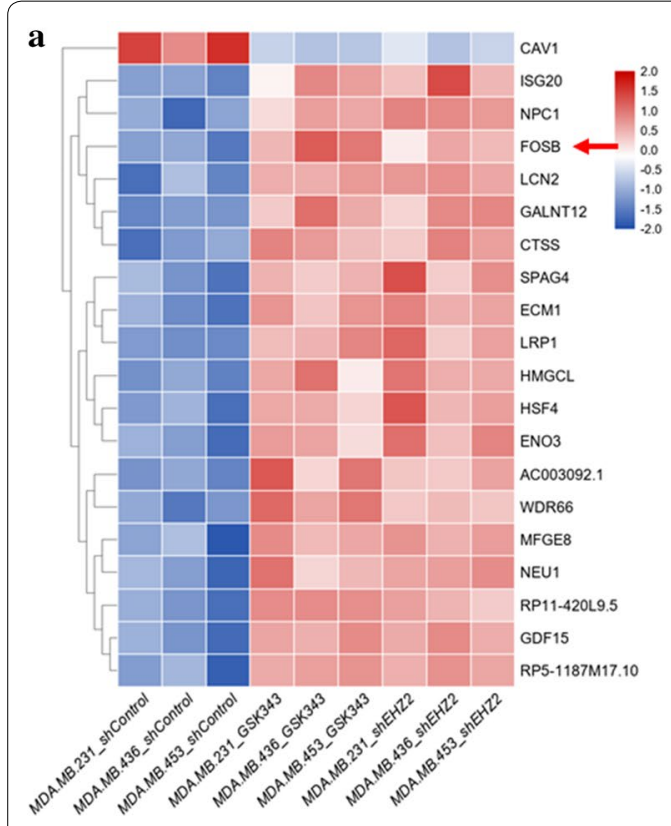

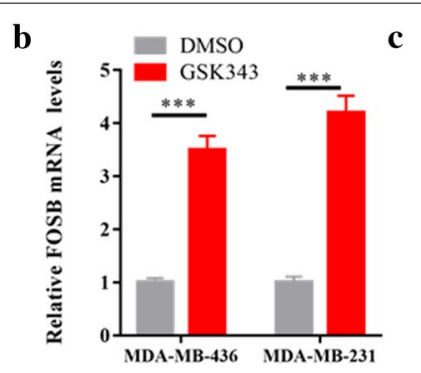

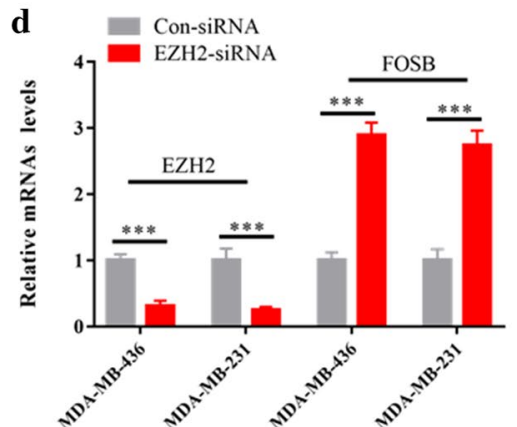

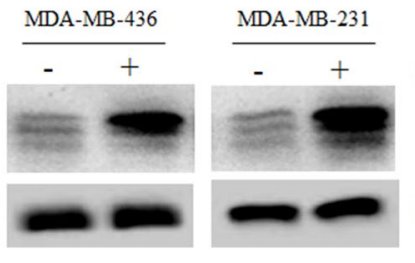

GSK343 FOSB GAPDH

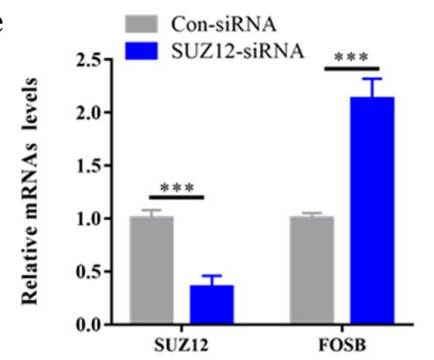

Fig. 1 Genetic ablation or pharmacological inhibition of EZH2 resulted in FOSB gene expression in TNBC cells. a Heatmap demonstrated the common differentially expressed genes in MDA-MB-231, MDA-MB-436 and MDA-MB-453 cells either treated with $2 \mu$ M GSK343, or stably transduced with shEHZ2, compared to untreated controls. $\mathbf{b}$ The mRNAs of FOSB in MDA-MB-231 and MDA-MB-436 cells with or without $2 \mu \mathrm{M}$ GSK343 treatment were determined by real-time PCR assay. c Immunoblot analysis of the indicated proteins in lysates from cells as in (a) with GAPDH as loading controls. $\mathbf{d}$ The mRNA levels of FOSB and EZH2 in MDA-MB-231 and MDA-MB-436 cells transfected with con-siRNA or siRNA against EZH2 were determined by real-time PCR assay. $\mathbf{e}$ The mRNAs of FOSB and SUZ12 in MDA-MB-231 cells transfected with con-siRNA or siRNA against SUZ12 were determined by real-time PCR assay 
As FOSB has been reported to be activated in several kinds of tumors, we hypothesized that FOSB may be a critical downstream target gene of EZH2 and mediate the function of EZH2is in TNBC. Consistent with the RNA-seq data, we found that GSK343 treatment significantly increased FOSB mRNA and protein expression in MDA-MB-231 and MDA-MB-436, two TNBC cell lines (Fig. 1b, c). Similarly, knockdown of EZH2 in both cells by siRNAs also promoted FOSB mRNA expression (Fig. 1d). Moreover, knockdown of the SUZ12 subunit of the PRC2 complex by siRNAs also induced FOSB upregulation [29] (Fig. 1e), suggesting FOSB is regulated by PRC2 complex.

\section{FOSB is downregulated and associated with better} relapse-free survival in TNBC

To understand the clinic relevance of FOSB, we firstly investigated its expression in TNBC. Analysis of Oncomine datasets revealed that FOSB was significantly downregulated in a variety of breast cancer samples (Additional file 1: Figure S1). Moreover, the downregulation of FOSB in TNBC was also confirmed in TCGA dataset (113 normal vs 115 TNBC, $P<0.0001)$ (Fig. 2a). By conducting gene set enrichment analysis (GSEA) of the RNA-seq data of those $115 \mathrm{TNBC}$, we found that HALLMARK_P53 and KRAS_SIGNALING_down pathways were significantly enriched, suggesting FOSB expression might associate with these tumor suppressing

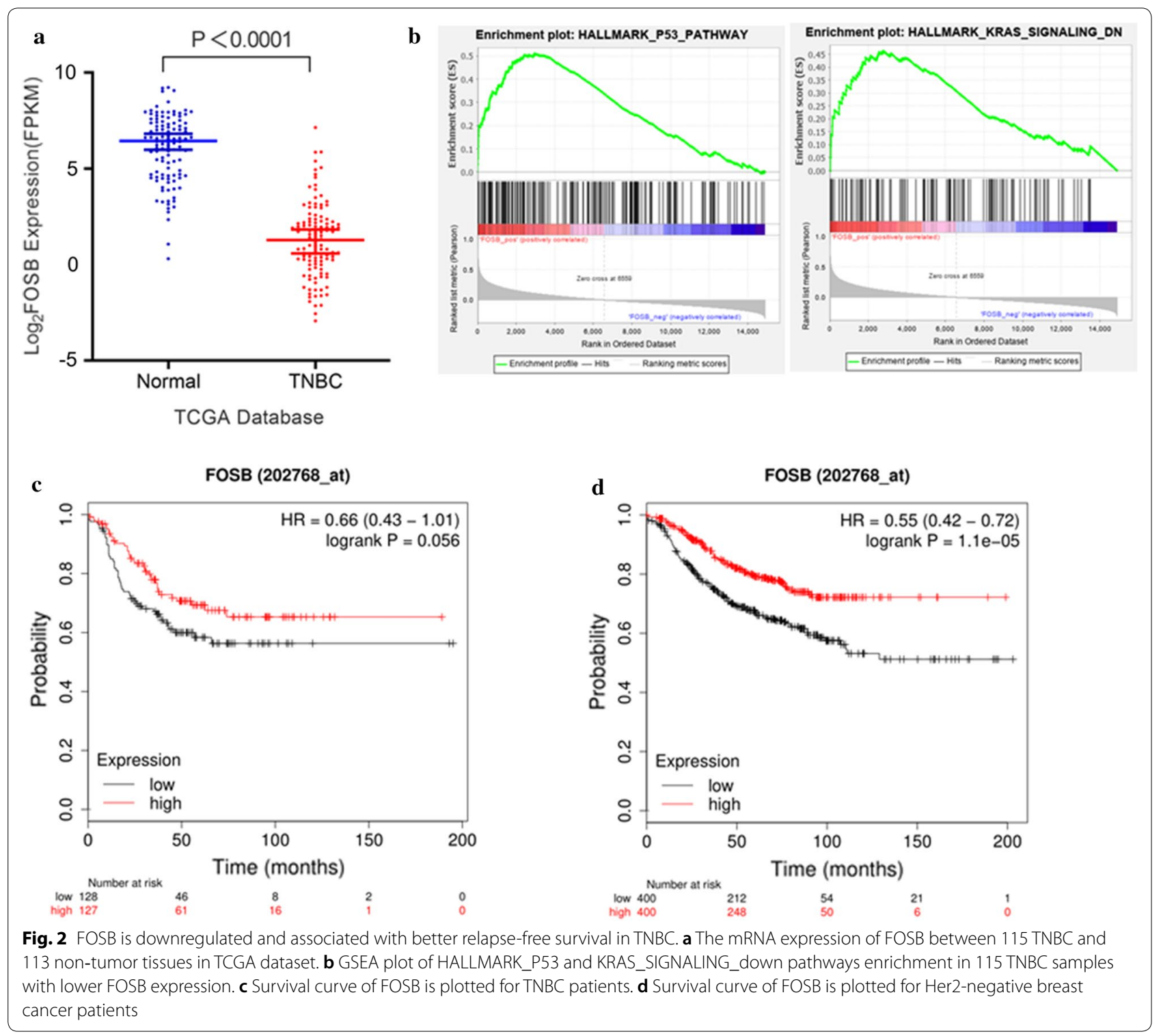


pathways (Fig. 2b). Next, we evaluated the prognosis value of FOSB in http://kmplot.com/analysis/. A survival analysis of the clinical breast cancer datasets indicated a strong correlation between higher FOSB expression and better relapse-free survival (RFS) ( $\mathrm{HR}=0.66,95 \%$ CI $0.43-1.41, P=0.056$ ) (Fig. 2c) in TNBC. Importantly, in Her2-negative breast cancer, a significant correlation between higher FOSB expression and better overallsurvival was also observed ( $\mathrm{HR}=0.55,95 \% \mathrm{CI} 0.42-0.72$, $P=1.1 \mathrm{e}-05$ ) (Fig. 2d). Together, these data suggested that aberrant expression of FOSB in TNBC is associated with better prognosis.

\section{H3K27me3 contributes to FOSB transcription silencing in TNBC}

EZH2-mediated H3K27me3 modification can further recruit methyltransferases to catalyze the methylation of CpG islands in the corresponding regions, so DNA methylation inhibitors could increase the expression of some PRC2 downstream genes expression [30]. To test this possibility, MDA-MB-231 and MDA-MB-436 cells were treated with a DNA methylation inhibitor 5-Aza-CdR
(5-Aza-2'-deoxycytidine). As expected, 5-Aza-CdR treatment significantly promoted FOSB mRNA and protein expression in both TNBC cells (Fig. 3a, b). To further determine whether EZH2-mediated H3K27me3 plays a direct role in FOSB gene silencing, we examined whether the H3K27me3 mark is enriched at the FOSB promoter. Chromatin immunoprecipitation (ChIP) assays showed abundant promoter binding of H3K27me3 in MDAMB-231 cells (Fig. 3c). Treatment with GSK343 could induce a global shift from trimethylation to acetylation at the H3K27 mark that to activate genes expression in the cells (Fig. 3d) [22]. Importantly, our ChIP assays confirmed the same changes at the FOSB promoter (Fig. 3e), which might contribute to the abundant reactivation of FOSB expression.

\section{$C / E B P \beta$ is required for FOSB gene epigenetic transcription activation}

We next investigated which transcriptional factors are involved in EZH2is-mediated FOSB gene reactivation. Analysis of the human FOSB promoter sequence

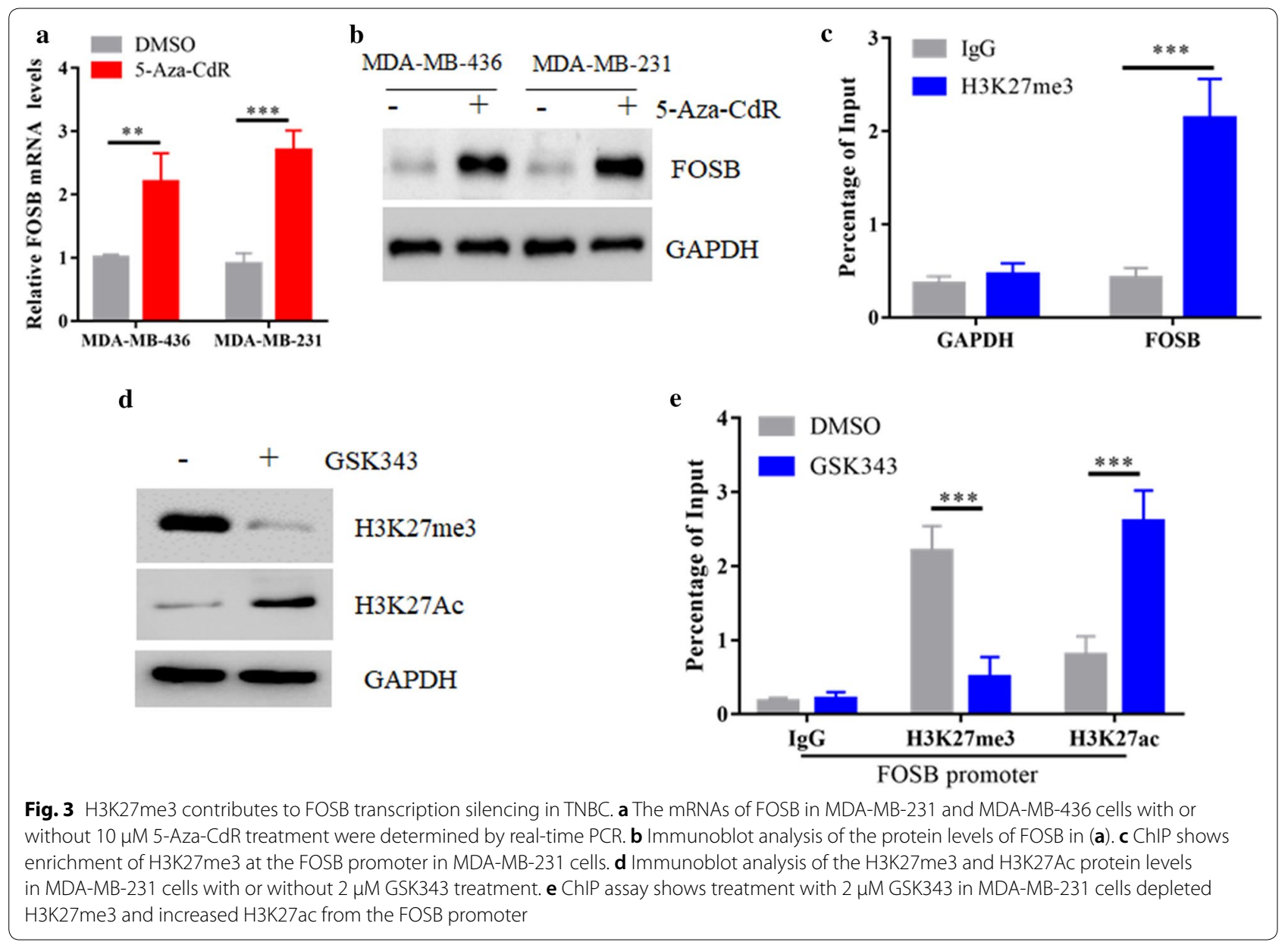


( -1000 to +1 bp relative to TSS) for potential transcription factor binding sites by the eukaryotic promoter database (EPD) predicted two binding sites for C/EBP $\beta$ (Fig. 4a). C/EBP $\beta$ can bind and recruit histone acetyltransferases p300 and CBP, which could catalyze H3K27 acetylation (H3K27ac) [31]. To test whether $\mathrm{C} / \mathrm{EBP} \beta$ is involved in FOSB reactivation, the expression of C/EBP $\beta$ was silenced by siRNAs and then treated GSK343. We found that knockdown of C/EBP $\beta$ significantly blocked GSK343-mediated FOSB expression (Fig. 4b). To further test whether C/EBP $\beta$ can directly activate FOSB transcription, we co-transfected C/EBP $\beta$ with a luciferase reporter vector under the control of the FOSB promoter $(-500$ to +1 bp of TSS) in 293 T cells. C/EBP $\beta$ could induce about threefold activation of luciferase activity (Fig. 4c), confirming that $\mathrm{C} / \mathrm{EBP} \beta$ can directly activate FOSB transcription. As two potential binding sites were existed in FOSB promoter region, we then determined which one is essential for FOSB expression. We found that mutation of either $\mathrm{C} / \mathrm{EBP} \beta$ binding site reduced, while mutation of both sites largely blocked the activity of FOSB promoter by C/EBP $\beta$ (Fig. 4d). Moreover, ChIP assays revealed that GSK343 treatment could promote abundant binding of $\mathrm{C} / \mathrm{EBP} \beta$ to the endogenous FOSB promoter (Fig. 4e). Taken together, these results indicate that epigenetic reactivation of FOSB expression by EZH2 inhibition mainly through two functional C/EBP $\beta$ binding sites in TNBC.

\section{FOSB exhibits tumor-suppressor functions in TNBC cells}

The GSEA results suggest FOSB might have a role in TNBC cells growth control (Fig. 2b). To this end, we firstly generated a FOSB overexpressing MDA-MB-436 cell line. The mRNA expression level of exogenous FOSB is about 3.5 times, which is close to the endogenous expression level in MDA-MB-436 cells induced by GSK343 (Fig. 5a). Overexpression of FOSB caused significantly decreased cell growth and colony formation ability (Fig. 5b, c). We further constructed a FOSB knock out (KO) MDA-MB-231 cell line using CRISPR-Cas9 technology to completely deplete FOSB gene (Fig. 5d), and found that FOSB KO cells showed increased proliferation and colony formation ability when compared with the control wild type (WT) cells (Fig. 5e, f). We next expanded our study to xenograft models to investigate whether FOSB affected TNBC cells proliferation in vivo. $\mathrm{BALB} / \mathrm{c}$ nude mice were subcutaneously injected with $1 \times 10^{7}$ FOSB WT or KO MDA-MB-231 cells for up to 4 weeks. Nude mice experiments confirmed that knock out of FOSB markedly increased MDA-MB-231 cells

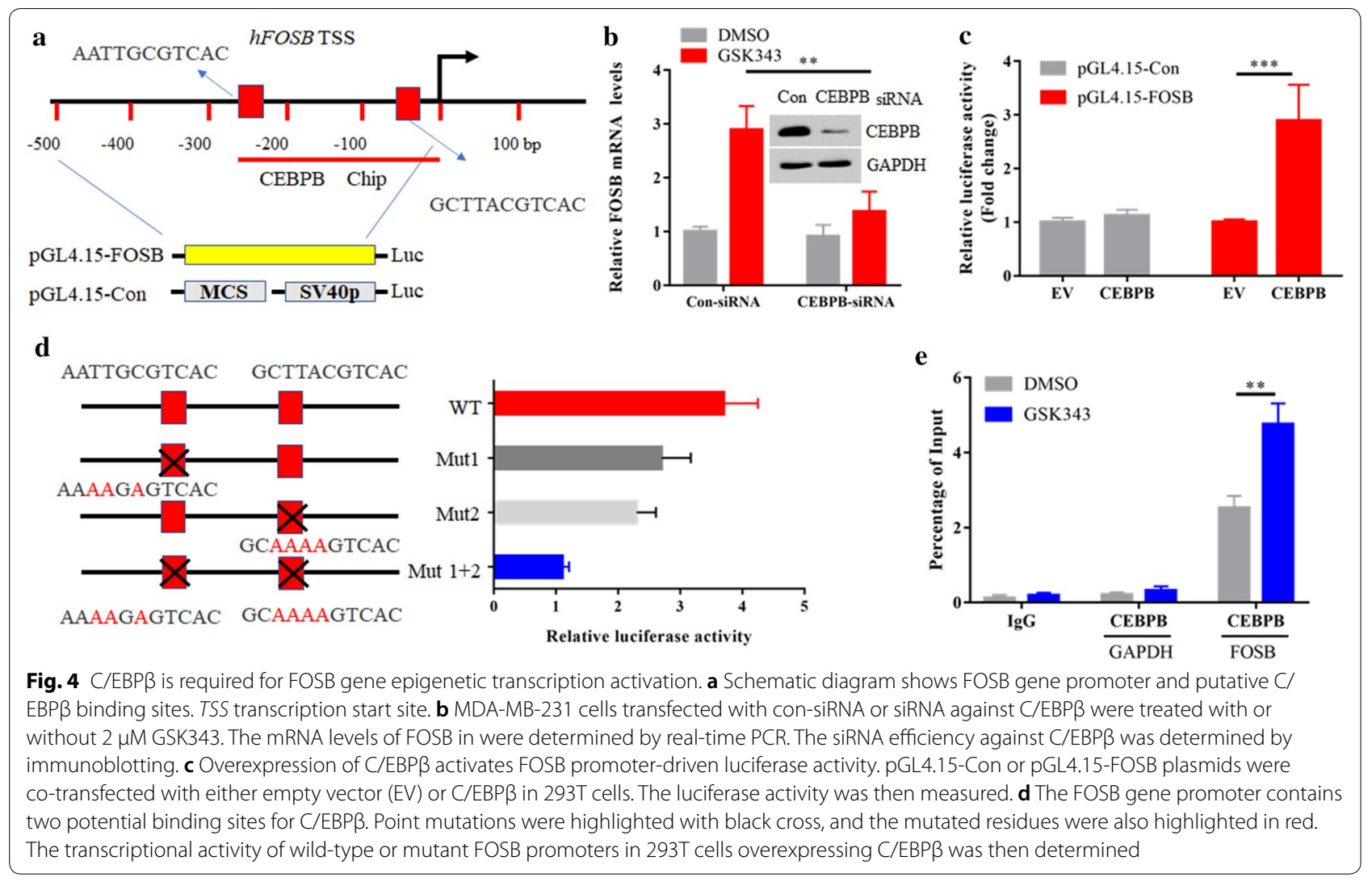



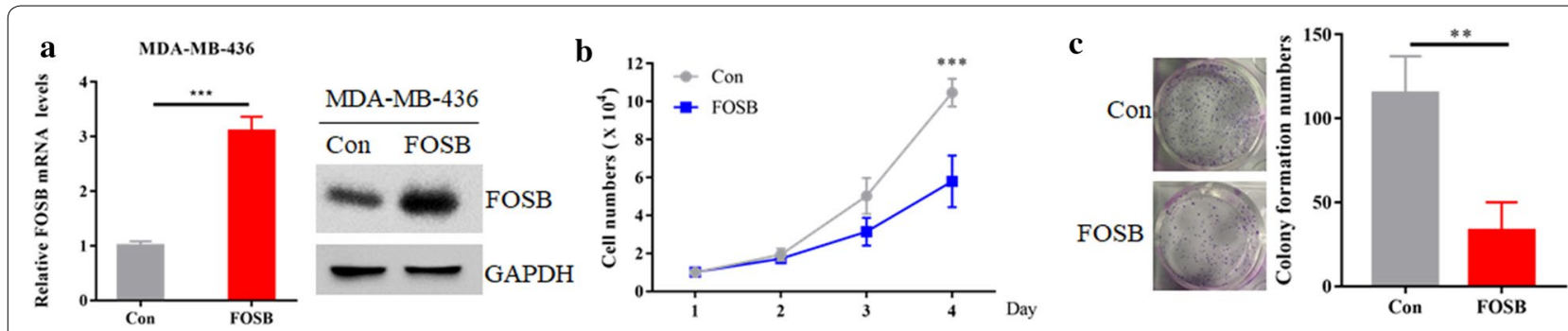

d
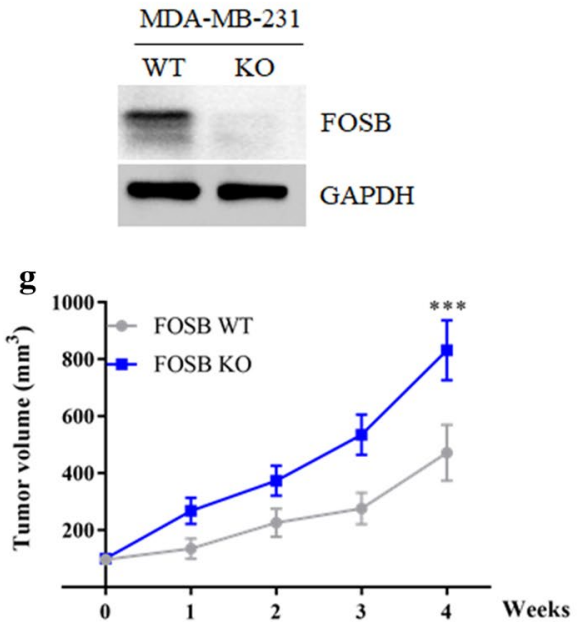

e

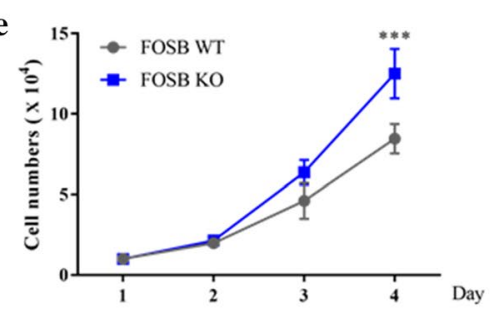

h

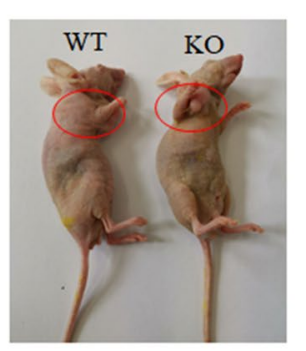

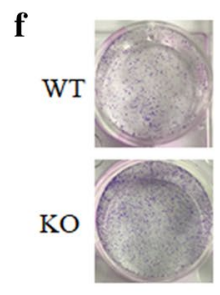

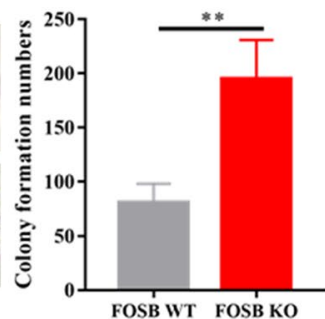

i

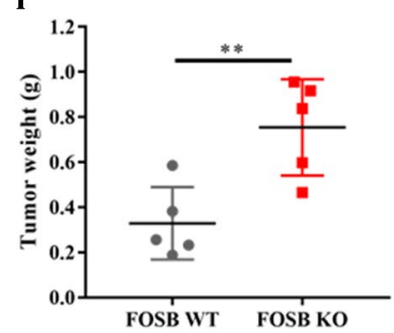

Fig. 5 FOSB exhibits tumor-suppressor functions in TNBC cells. a The mRNA and protein levels of FOSB from MDA-MB-231 cells stable expression of pcDNA3(Con) or pcDNA3-FOSB were detected by real-time PCR assay and western blot, respectively. $\mathbf{b}$ The cell growth curve of MDA-MB-231 cells stable expression of pcDNA3(Con) or pcDNA3-FOSB. c Clonogenic assay of MDA-MB-231 cells stable expression of pcDNA3(Con) or pcDNA3-FOSB. 1000 cells were seeded into each well of a six-well plate, and cultured for about 2 weeks. Cells were stained with crystal violet and then counted. d FOSB KO MDA-MB-231 cells were generated by CRISPR assay and detected by western blot. e The cell growth curve of FOSB WT and KO MDA-MB-231 cells. $\mathbf{f}$ Clonogenic assay of FOSBWT and KO MDA-MB-231 cells. 1000 cells were seeded into each well of a six-well plate, and cultured for about 2 weeks. Cells were stained with crystal violet and then counted. $\mathbf{g}$ Each nude mouse was subcutaneously injected with $1 \times 10^{7}$ FOSB WT and KO MDA-MB-231 cells for about 4 weeks. Tumour growth was measured using a caliper at the indicated times after injection. $\mathrm{n}=5$ for each group. ${ }^{* *} \mathrm{P}<0.001$. $\mathbf{h}$ The image shows representative tumor-bearing mice for each group. $\mathbf{i}$ Mice tumor weights in each group were measured after mice were sacrificed. ${ }^{* *} \mathrm{P}<0.01$

tumor growth (Fig. 5g-i). Thus, these data indicated that FOSB played a tumor-suppressor role in the regulation of TNBC cells proliferation both in vitro and in vivo.

\section{EZH2is reduce TNBC cell growth in a FOSB-dependent fashion}

It has been reported that lower concentrations of GSK343 $(0.5 \mu \mathrm{M}$ and $2 \mu \mathrm{M})$ consistently inhibited the proliferation of TNBC cell lines [28]. To test whether FOSB affected the sensitivity of TNBC cells to GSK343 treatment, we treated FOSB WT and KO MDA-MB-231 cells with $2 \mu \mathrm{M}$ GSK343. We found that knock out of FOSB significantly abrogated the antiproliferative effect of GSK343 (Fig. 6a). The similar results were also observed by using an another EZH2 inhibitor EPZ-6438 (Fig. 6b), suggesting that EZH2is-depended TNBC cell growth retardant required FOSB reactivation. It has been reported that depletion of p53 significantly affected the anti-proliferation role of EZH2is in TNBC cells [28]. We found that the mRNA levels of p53 and its downstream target CDKN1A (p21 ${ }^{\mathrm{CIP} 1}$ ) were significantly decreased in FOSB KO MDA-MB-231 cells (Fig. 6c). Moreover, GSK343 treatment failed to induced the expression of p53 in FOSB KO cells (Fig. 6d). Together, these data indicated that FOSB-mediated p53 expression is required for the antiproliferative effect of EZH2is in TNBC cells.

\section{Discussion}

In the present study, we report that EZH2-mediated epigenetic inactivation of FOSB in TNBC cells and FOSB expression is correlated with better relapse-free survival in TNBC tissues. In line with these observations, we showed that genetic inhibition of FOSB promoted TNBC cells proliferation in vitro, and thus tumor progression in 

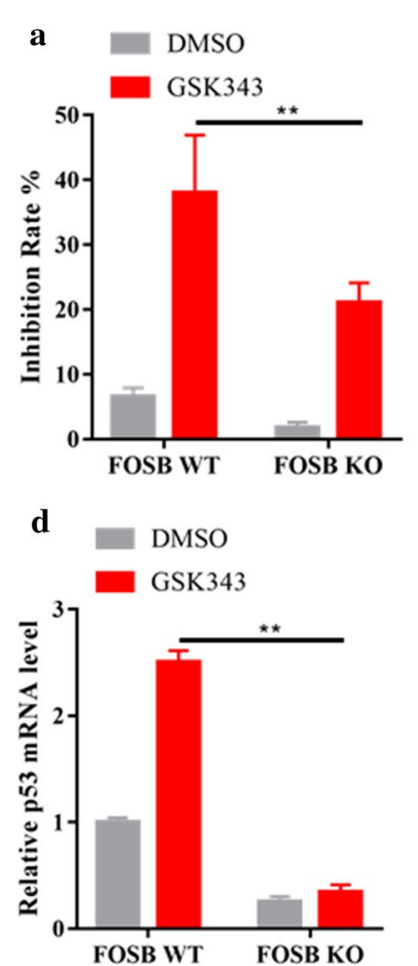
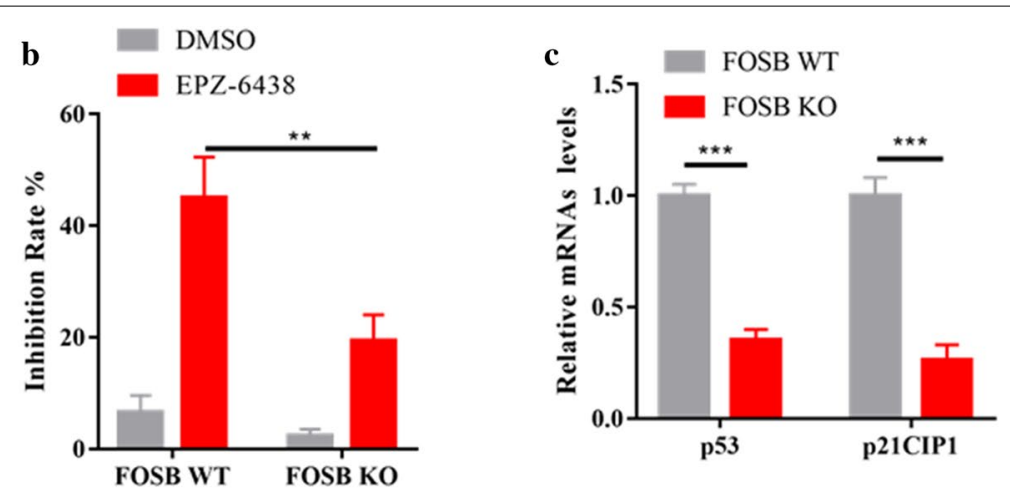

e

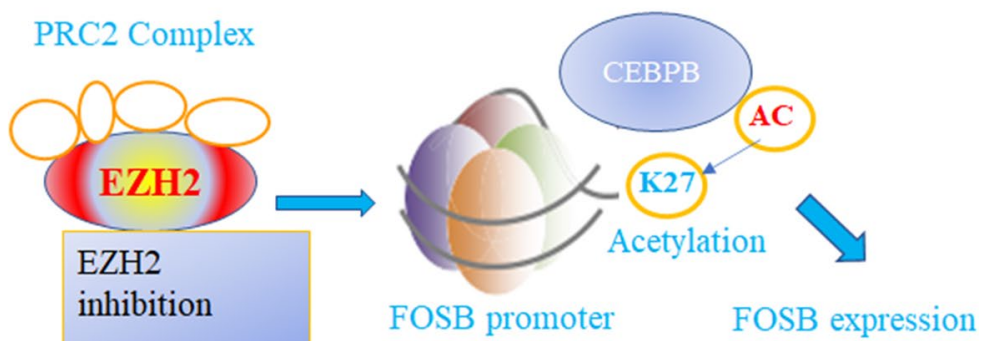

Fig. 6 EZH2is reduce TNBC cell growth in a FOSB-dependent fashion. a Cell growth assay. FOSB WT and KO MDA-MB-231 cells were exposed to $2 \mu$ M GSK343 for 6 days. Cell growth inhibition rate was measured by CCK8 assay. $n=3$ replicates. $\mathbf{b}$ FOSB WT and KO MDA-MB-231 cells were exposed to $5 \mu \mathrm{M}$ EPZ-6438 for 6 days. Cell growth inhibition rate was measured by CCK8 assay. $n=3$ replicates. $\mathbf{c}$ The mRNA levels of p53 and P2 $1^{\mathrm{CIP} 1}$ in FOSB WT and KO MDA-MB-231 cells were determined by real-time PCR assay. $\mathbf{d}$ FOSB WT and KO MDA-MB-231 cells were treated with or without $2 \mu \mathrm{M}$ GSK343. The mRNA levels of p53 in determined by real-time PCR assay. e Proposed model for the action of EZH2is. EZH2is promoted the shift from H3K27me3 to H3K27ac at the FOSB promoter, and recruited the transcription factor C/EBP $\beta$ to activate FOSB gene transcription

subcutaneously transplanted TNBC mice, indicating a critical role of FOSB in the regulation of TNBC progress. After EZH2is treatment, FOSB expression was significantly increased, a process required the involvement of the transcriptional factor $\mathrm{C} / \mathrm{EBP} \beta$. Indeed, two potential $\mathrm{C} / \mathrm{EBP} \beta$ binding sites were located in the promoter region of FOSB gene. Thus, our data clearly showed that upon $\mathrm{EZH} 2$ is treatment, the transition from H3K27me3 to $\mathrm{H} 3 \mathrm{~K} 27 \mathrm{ac}$ at the promoter of FOSB gene recruited $\mathrm{C} / \mathrm{EBP} \beta$ to facilitate FOSB gene expression in TNBC. Importantly, we found that FOSB-depleted TNBC cells were resistant to $\mathrm{EZH} 2$ is treatment, implying loss of FOSB might confer drug resistance to EZH2is. Thus, our results suggested that EZH2-mediated epigenetic inactivation of FOSB promoted TNBC expression (Fig. 6e), demonstrating that reactivation of FOSB expression underlies the anti-TNBC action of EZH2is.

There are still some issues to be clarified. For instance, the sample size in our present study is relatively small. Further studies using larger clinic TNBC samples are still needed to confirm the relationship between EZH2 and FOSB. Moreover, it might be interested and important to study the relationship between EZH2 and other AP-1 family members in the future.

In addition to its known roles in histone modification and transcriptional regulation, EZH2 has been reported to methylate non-histone substrates. EZH2 binds and methylates STAT3 to promote tumorigenicity of glioblastoma [32]. EZH2 can bind and methylate androgen receptor (AR) to modulate $A R$ recruitment to the sites bound by both AR and EZH2 [33]. EZH2 could also promote monomethylation of RORa to mark it for degradation by the DCAF1/DDB1/CUL4 E3 ubiquitin ligase complex [34]. Although we found that EZH2is could promote the shift from H3K27me3 to H3K27ac at FOSB promoter to facilitate FOSB gene expression, we could not exclude the possibility that EZH2 might also bind and methylate other non-histone substrate to regulate FOSB expression.

\section{Conclusion}

Together, our in vivo and in vitro results demonstrate that targeting of EZH2 in TNBC might cause epigenetic reactivation of FOSB genes and activation of p53 pathway, 
providing a novel mechanism of EZH2is treatment in TNBC. Our work shows that FOSB might be the major effector of EZH2is in TNBC, and that the therapeutic effects of EZH2is will be lost in the absence of FOSB. Thus, we can predict that the FOSB negative TNBC will not respond to EZH2is treatment and lead to therapeutic resistance. The future studies will need to include strategies to counter emergence of EZH2is resistance in FOSB negative TNBC patients.

\section{Supplementary information}

Supplementary information accompanies this paper at https://doi. org/10.1186/s12935-020-01260-5.

Additional file 1: Figure S1. FOSB was significantly downregulated in a variety of breast cancer samples in Oncomine datasets.

\section{Abbreviations}

H3K27me3: Histone 3 trimethylated on lysine 27; EZH2i: EZH2 inhibitor; RFS: Relapse-free survival; TNBC: Triple-negative breast cancer; Chip: Chromatin immunoprecipitation; ER: Estrogen receptor; PR: Progesterone receptor; HER2: The human epidermal growth factor receptor 2; GCB: Germinal center B cell; DLBCL: Diffuse large cell B-cell lymphomas; FL: Follicular lymphomas.

\section{Acknowledgements}

Not applicable.

\section{Authors' contributions}

$R Z, X L$ and $H X$ participated in the design of the study and performed the experiments. $R Z, X L$ and $Z L$ performed the primary experience. $Y W$ and $H Z$ performed the statistical analyses. $\mathrm{HX}$ was the major contributor to the design of this study and wrote the manuscript. All authors read and approved the final manuscript.

\section{Funding}

This project is funded by the Liaoning Cancer Hospital and Institute.

\section{Availability of data and materials}

The GEO data we used is GSE112378.

\section{Ethics approval and consent to participate}

This study was approved by the Animal Care and Use Committee of Liaoning Cancer Hospital and Institute.

\section{Consent for publication}

All listed authors have actively participated in the study and have read and approved the submitted manuscript.

\section{Competing interests}

The authors declare that they have no competing interests.

Received: 5 March 2020 Accepted: 12 May 2020

Published online: 19 May 2020

\section{References}

1. Sorlie T, Perou CM, Tibshirani R, Aas T, Geisler S, Johnsen H, Hastie T, Eisen $M B$, van de Rijn $M$, Jeffrey SS, et al. Gene expression patterns of breast carcinomas distinguish tumor subclasses with clinical implications. Proc Natl Acad Sci USA. 2001;98(19):10869-74.

2. Perou CM, Sorlie T, Eisen MB, van de Rijn M, Jeffrey SS, Rees CA, Pollack JR, Ross DT, Johnsen H, Akslen LA, et al. Molecular portraits of human breast tumours. Nature. 2000;406(6797):747-52
3. Bianchini G, Balko JM, Mayer IA, Sanders ME, Gianni L. Triple-negative breast cancer: challenges and opportunities of a heterogeneous disease. Nat Rev Clin Oncol. 2016;13(11):674-90.

4. Rouzier R, Perou CM, Symmans WF, Ibrahim N, Cristofanilli M, Anderson K, Hess KR, Stec J, Ayers M, Wagner P, et al. Breast cancer molecular subtypes respond differently to preoperative chemotherapy. Clin Cancer Res. 2005;11(16):5678-85.

5. Muhammad N, Steele R, Isbell TS, Philips N, Ray RB. Bitter melon extract inhibits breast cancer growth in preclinical model by inducing autophagic cell death. Oncotarget. 2017;8(39):66226-36.

6. Muhammad N, Bhattacharya S, Steele R, Ray RB. Anti-miR-203 suppresses ER-positive breast cancer growth and stemness by targeting SOCS3. Oncotarget. 2016;7(36):58595-605.

7. Mohammad N, Singh SV, Malvi P, Chaube B, Athavale D, Vanuopadath M, Nair SS, Nair B, Bhat MK. Strategy to enhance efficacy of doxorubicin in solid tumor cells by methyl-beta-cyclodextrin: involvement of p53 and Fas receptor ligand complex. Sci Rep. 2015;5:11853.

8. SuY, Hopfinger NR, Nguyen TD, Pogash TJ, Santucci-Pereira J, Russo J. Epigenetic reprogramming of epithelial mesenchymal transition in triple negative breast cancer cells with DNA methyltransferase and histone deacetylase inhibitors. J Exp Clin Cancer Res. 2018;37(1):314.

9. Shu S, Lin CY, He HH, Witwicki RM, Tabassum DP, Roberts JM, Janiszewska M, Huh SJ, Liang Y, Ryan J, et al. Response and resistance to BET bromodomain inhibitors in triple-negative breast cancer. Nature. 2016;529(7586):413-7.

10. Comet I, Riising EM, Leblanc B, Helin K. Maintaining cell identity: PRC2-mediated regulation of transcription and cancer. Nat Rev Cancer. 2016;16(12):803-10.

11. Laugesen A, Hojfeldt JW, Helin K. Molecular mechanisms directing PRC2 recruitment and H3K27 methylation. Mol Cell. 2019;74(1):8-18.

12. Varambally S, Dhanasekaran SM, Zhou M, Barrette TR, Kumar-Sinha C Sanda MG, Ghosh D, Pienta KJ, Sewalt RG, Otte AP, et al. The polycomb group protein $\mathrm{EZH} 2$ is involved in progression of prostate cancer. Nature. 2002;419(6907):624-9.

13. Bachmann IM, Halvorsen OJ, Collett K, Stefansson IM, Straume O, Haukaas SA, Salvesen HB, Otte AP, Akslen LA. EZH2 expression is associated with high proliferation rate and aggressive tumor subgroups in cutaneous melanoma and cancers of the endometrium, prostate, and breast. J Clin Oncol. 2006;24(2):268-73.

14. Donaldson-Collier MC, Sungalee S, Zufferey M, Tavernari D, Katanayeva N, Battistello E, Mina M, Douglass KM, Rey T, Raynaud F, et al. EZH2 oncogenic mutations drive epigenetic, transcriptional, and structural changes within chromatin domains. Nat Genet. 2019;51(3):517-28.

15. Kleer CG, Cao Q, Varambally S, Shen R, Ota I, Tomlins SA, Ghosh D, Sewalt $R G$, Otte AP, Hayes DF, et al. EZH2 is a marker of aggressive breast cancer and promotes neoplastic transformation of breast epithelial cells. Proc Natl Acad Sci USA. 2003;100(20):11606-11.

16. Lohr JG, Stojanov P, Lawrence MS, Auclair D, Chapuy B, Sougnez C, CruzGordillo P, Knoechel B, Asmann YW, Slager SL, et al. Discovery and prioritization of somatic mutations in diffuse large B-cell lymphoma (DLBCL) by whole-exome sequencing. Proc Natl Acad Sci USA. 2012;109(10):3879-84

17. Morin RD, Johnson NA, Severson TM, Mungall AJ, An J, Goya R, Paul JE, Boyle M, Woolcock BW, Kuchenbauer F, et al. Somatic mutations altering EZH2 (Tyr641) in follicular and diffuse large B-cell lymphomas of germinal-center origin. Nat Genet. 2010;42(2):181-5.

18. Soumyanarayanan U, Dymock BW. Recently discovered EZH2 and EHMT2 (G9a) inhibitors. Future Med Chem. 2016;8(13):1635-54.

19. Kim KH, Roberts CW. Targeting EZH2 in cancer. Nat Med. 2016;22(2):128-34.

20. Knutson SK, Kawano S, Minoshima Y, Warholic NM, Huang KC, Xiao Y, Kadowaki T, Uesugi M, Kuznetsov G, Kumar N, et al. Selective inhibition of EZH2 by EPZ-6438 leads to potent antitumor activity in EZH2-mutant non-Hodgkin lymphoma. Mol Cancer Ther. 2014;13(4):842-54.

21. Verma SK, Tian X, LaFrance LV, Duquenne C, Suarez DP, Newlander KA, Romeril SP, Burgess JL, Grant SW, Brackley JA, et al. Identification of potent, selective, cell-active inhibitors of the histone lysine methyltransferase EZH2. ACS Med Chem Lett. 2012;3(12):1091-6.

22. Huang $X$, Yan J, Zhang M, Wang Y, Chen Y, Fu X, Wei R, Zheng XL, Liu Z, Zhang $X$, et al. Targeting epigenetic crosstalk as a therapeutic strategy for EZH2-aberrant solid tumors. Cell. 2018;175(1):186-199.e119. 
23. Jochum W, Passegue E, Wagner EF. AP-1 in mouse development and tumorigenesis. Oncogene. 2001;20(19):2401-12.

24. Renaud SJ, Kubota K, Rumi MA, Soares MJ. The FOS transcription factor family differentially controls trophoblast migration and invasion. J Biol Chem. 2014;289(8):5025-39.

25. Milde-Langosch $\mathrm{K}$. The Fos family of transcription factors and their role in tumourigenesis. Eur J Cancer. 2005;41(16):2449-61.

26. Muhammad N, Bhattacharya S, Steele R, Phillips N, Ray RB. Involvement of c-Fos in the promotion of cancer stem-like cell properties in head and neck squamous cell carcinoma. Clin Cancer Res. 2017;23(12):3120-8.

27. Ran FA, Hsu PD, Wright J, Agarwala V, Scott DA, Zhang F. Genome engineering using the CRISPR-Cas9 system. Nat Protoc. 2013;8(11):2281-308.

28. Yu Y, Qi J, Xiong J, Jiang L, Cui D, He J, Chen P, Li L, Wu C, Ma T, et al. Epigenetic co-deregulation of EZH2/TET1 is a senescence-countering, actionable vulnerability in triple-negative breast cancer. Theranostics. 2019;9(3):761-77.

29. Lee W, Teckie S, Wiesner T, Ran L, Prieto Granada CN, Lin M, Zhu S, Cao $Z$, Liang $Y$, Sboner $A$, et al. PRC2 is recurrently inactivated through EED or SUZ12 loss in malignant peripheral nerve sheath tumors. Nat Genet. 2014:46(11):1227-32.

30. Vire E, Brenner C, Deplus R, Blanchon L, Fraga M, Didelot C, Morey L, Van Eynde A, Bernard D, Vanderwinden JM, et al. The Polycomb group protein EZH2 directly controls DNA methylation. Nature. 2006;439(7078):871-4.
31. Schwartz C, Beck K, Mink S, Schmolke M, Budde B, Wenning D, Klempnauer $\mathrm{KH}$. Recruitment of p300 by C/EBPbeta triggers phosphorylation of p300 and modulates coactivator activity. EMBO J. 2003;22(4):882-92.

32. Kim E, Kim M, Woo DH, Shin Y, Shin J, Chang N, Oh YT, Kim H, Rheey J, Nakano I, et al. Phosphorylation of EZH2 activates STAT3 signaling via STAT3 methylation and promotes tumorigenicity of glioblastoma stemlike cells. Cancer Cell. 2013;23(6):839-52.

33. Yu J, Yu J, Mani RS, Cao Q, Brenner CJ, Cao X, Wang X, Wu L, Li J, Hu M, et al. An integrated network of androgen receptor, polycomb, and TMPRSS2-ERG gene fusions in prostate cancer progression. Cancer Cell. 2010;17(5):443-54.

34. Lee JM, Lee JS, Kim H, Kim K, Park H, Kim JY, Lee SH, Kim IS, Kim J, Lee M, et al. EZH2 generates a methyl degron that is recognized by the DCAF1/ DDB1/CUL4 E3 ubiquitin ligase complex. Mol Cell. 2012;48(4):572-86.

\section{Publisher's Note}

Springer Nature remains neutral with regard to jurisdictional claims in published maps and institutional affiliations.
Ready to submit your research? Choose BMC and benefit from:

- fast, convenient online submission

- thorough peer review by experienced researchers in your field

- rapid publication on acceptance

- support for research data, including large and complex data types

- gold Open Access which fosters wider collaboration and increased citations

- maximum visibility for your research: over $100 \mathrm{M}$ website views per year

At BMC, research is always in progress.

Learn more biomedcentral.com/submissions 